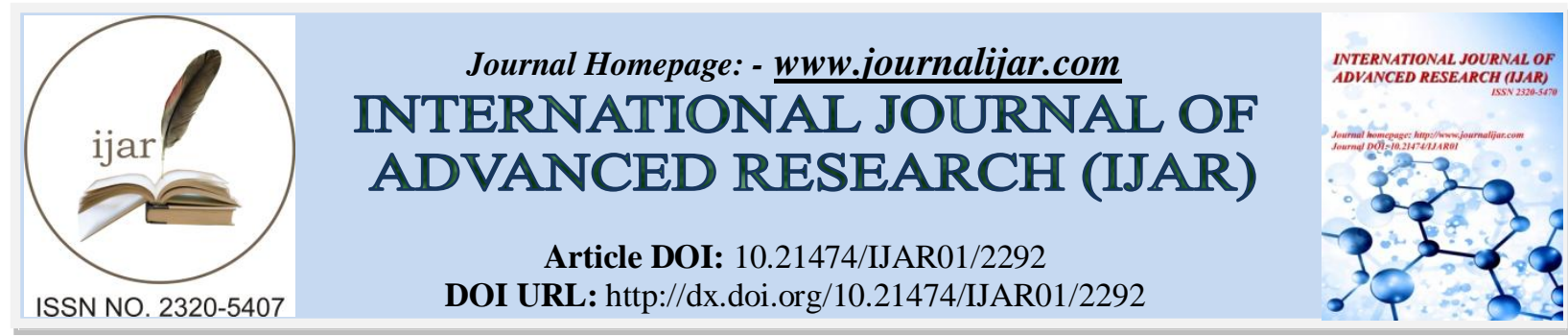

RESEARCH ARTICLE

\title{
ALTERATION IN IMMUNE RESPONSE PATTERN AT T-CELL LEVEL DURING HYPER- THYROIDISM AND HYPOTHYROIDISM CLINICAL CONDITION OF AUTO IMMUNE DISORDER IN CONTEXT TO HEALTHY CONTROL.
}

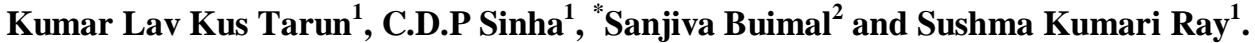 \\ 1. PG Dept. Of Biotechnology, Magadh University, Bodhgaya, Bihar. \\ 2. Dept. Of Immunology, Rajendra Memorial Research Institute of Medical Sciences, Agamkuan, Patna.
}

\section{Manuscript Info}

Manuscript History

Received: 29 September 2016

Final Accepted: 30 October 2016

Published: November 2016

\section{Abstract}

The risk of infection in many auto immune diseases especially in thyroid patients has posed a major challenge in control programmes. We undertook this study to identify the high risk groups vulnerable to thyroid disease in the State of Bihar, India. Further, immunological responses were also evaluated in these patients showing high and moderate level of TSH for thyroid to see the immune impairment associated with CD4 and CD8 T cell count. Lymphocytes from these patients were further analyzed for Th1( Interferon gamma) and Th2( interleukin-4) intracellular cytokines using the Cell Quest software on a FACS Calibur flow cytometer. Of the 90 thyroid -positive individuals, fifteen(15) who had shown a higher TSH value( 48.55 or above) were categorised as thyroid patients under hyperthyroidism whereas fifteen(15) patients who had shown a lower TSH value( 0.22- 0.02) were categorised as thyroid patients under hypothyroidism patients. The cellular immune responses were evaluated in 15 Indian Thyroid patients with hyper thyrodism category $(n=15)$ and 15 thyroid patients under hypo-thyroidism category. Further, such immunological responses were also evaluated in VL patients( $n=10)$ patients, HIV cases $(\mathrm{n}=10)$ and 10 apparently healthy controls to see the immune impairment especially associated with Thyroid disease. A very high absolute CD3 cell count was observed in thyroid cases under both category segregated based on TSH value compared to HIV and VL patients or controls. No significant difference was observed for CD4 cell count in both categories of thyroid patients, however a reduction in CD4 count was very much apparent than healthy control but it was similar an identical reduced CD4 count observed in VL cases. The CD8+ T cell counts showed varied patterns in higher TSH category of thyroid patients and recorded a higher value whereas it was lower in the thyroid patients showing lower TSH value. All hyper thyrodism patients had higher interferon-gamma( IFN- $\gamma$ ) produced in culture supernants compared to Thyroid patients with low TSH range, VL, HIV and healthy control. Apart from it, although level of IL-4 was distinctly higher than control, we observed a lower production of interleukin-4 in thyroid patients compared to VL cases and HIV but thyroid patients under hyper-thyroidism category shown with the 
elevation in the levels of IL-4 which was about two-fold higher in the thyroid cases with hypo-thyroidism state. In summary, a different immune response for CD8 pattern, IFN- $\gamma$, and IL-4 was noticed in thyroid patients with hyper thyroid state. Unlike to other diseases investigated ( VL and HIV), there was a significant increase in absolute T-cell count ( CD3 + T cells) under both category of thyroid disease. Further studies need to be done to see the effect of therapy on immunological parameters in these patients. Further studies will be needed to clarify the exact role of peripheral lymphocytes in thyroid patients and whether they could provide a reliable marker of thyroid immune involvement.

Copy Right, IJAR, 2016,. All rights reserved.

\section{Introduction:-}

The diseases of the human thyroid gland are goiter (diffuse or nodular), hyperthyroidism, hypothyroidism, autoimmune thyroiditis, and neoplasm [Kumar et al, 2010]. The thyroiditis types cause inflammation of thyroid tissue and can release preformed hormone from the colloid space, causing thyrotoxicosis, which is transient and followed by recovery or development of hypothyroidism. These conditions result by reactivity to self-thyroid antigens which are expressed as distinctive inflammatory or antireceptor autoimmune diseases [Weetman and A. M. McGregor, 1994; Eguchi,1995]. The incidence is 0.3 to 1.5 per 1000 persons per year, and it is 4 to 10 times more common in women than in men [Huber et al, 2008, Iddah et al,2013, Canaris et al,2013]. Susceptibility to the disease is determined by a combination of immune mechanism, genetics, and environmental (iodine, infection, and stress) and constitutional factors.

All forms of thyroid autoimmunity are associated with a lymphocytic infiltrate in the thyroid. These lymphocytes are largely responsible for generating both $\mathrm{T}$ - and B-cell-mediated auto reactivity. Other sites such as thyroid draining lymph nodes and bone marrow may also contain thyroid autoreactive lymphocytes in AITD. The initial autoimmune response by $\mathrm{CD} 4+\mathrm{T}$ cells appears to upregulate the secretion of interferon-gamma resulting in enhancing the expression of MHC II molecules on thyrocytes. This most likely triggers expansion of autoreactive T cells and gives rise to the characteristic inflammatory response, and as the disease progresses, thyrocytes are targeted for apoptosis resulting in hypothyroidism. Another contributing factor to the observed hypothyroidism in Hashimoto's thyroiditis patients could be the circulating TSH inhibitory antibodies. Graves' disease on the other hand represents the other end of spectrum wherein the patients suffer from hyperthyroidism. The activation of thyroid specific CD4+ T cells leads to the recruitment of autoreactive B cells and the mounting of thyroid stimulatory immune response via antithyroid antibodies [McLachlan and B. Rapoport, 1992].

It is anticipated that the infection by self-thyroid antigens in thyroid patients might cause reciprocal enhanced immunological disturbances. However, data on prospective distribution on CD3+CD4+ and CD8+ cell count and cytokine pattern elicited by Th1 and Th2 functional subpopulation of T cells in thyroid cases are relatively scarce. We undertook this study to identify the high risk groups vulnerable to thyroid disease in the State of Bihar, India. Further, immunological responses were also evaluated in these patients showing high and moderate level of TSH for thyroid to see the immune impairment associated with CD4 and CD8 T cell count. Lymphocytes from these patients were further analyzed for Th1( Interferon gamma) and Th2( interleukin-4) intracellular cytokines using the Cell Quest software on a FACS Calibur flow cytometer.

\section{Material and Methods:-}

Subjects: A total of 60 subjects (aged between 35 to 50 years) of both sexes were studied after obtaining their informed consent. It included 15 patients with acute Thyroid condition from Gaya, Bihar, 15 patients of thyroid with moderate or a low TSH level, 10 patients of acute VL ( under a collaboration with Dr Sanjiva Bimal, RMRIMS, ICMR, Patna) 10 patients infected with HIV and reported for screening at RMRIMS, Patna and 10 apparently healthy individuals who represented Gaya District areas from Bihar. The level of TSH hormone was estimated from suspected thyroid patients.Based on such results, cases who had shown a higher TSH value( 48.55 or above) were categorised as thyroid patients under hyperthyroidism whereas fifteen(15) patients who had shown a lower TSH value( $0.22-0.02)$ were categorised as thyroid patients under hypothyroidism patients 
Suspected patients in the VL group under clinical and expert supervision of RMRI group were selected who had presented characteristic signs and symptoms of VL infection and diagnosis was confirmed by presence of $L$. donovani in the Giemsa stained spleen aspirate and positive serology (DAT).

The HIV samples in which diagnosis was confirmed by HIV unit at RMRIMS by HIV EIA (Lab system Kit, Finland) and rapid test (NEVA HIV, Cadila, India) were also enrolled in study. All the healthy controls were negative for all disease symptoms and matched the diseased cases by sex and age ( \pm 2years).

\section{Immunological Investigations:-}

Subsequent experiments were performed using immunoflourescent staining for CD4 and CD8 countand detection of level of interferon- $\gamma(\mathrm{IFN} \gamma)$ and interleukin-4 (IL4) cytokines in culture supernatants. For CD4 and CD8 count, whole blood was collected in EDTA-containing vacutainer tubes (Becton Dickinson, Rutherford, USA). The blood was stained with $20 \mu \mathrm{l}$ fluorescent BD Tri Test monoclonalantibodies specific for CD4/CD8/CD3 conjugated to (FITC), (PE) and per (CP) respectively and incubated for $30 \mathrm{~min}$ at room temperature in a dark area. After staining, cells were suspended in $450 \mu \mathrm{l}$ of $1 \mathrm{x}$ Lysing solution and incubated at room temperature for $10 \mathrm{~min}$ in dark. Cells were examined for fluorescence in a FACS-calibur (BD, San Diego, USA). Flowcytometry data were evaluated on Cell Quest software. For cytokine detection, whole blood was collected in vacutainer tubes containing heparin. Peripheral blood mononuclear cells (PBMC) were separated by Ficoll-Hypaque density gradient centrifugation, washed in phosphate buffered saline, $p \mathrm{H}$ 7.2-7.4 and resuspended in (RPMI) 1640 medium with 10 per cent foetal calf serum, $100 \mathrm{U} / \mathrm{ml}$ of penicillin and $100 \mu \mathrm{g} / \mathrm{ml}$ of streptomycin. These cells were cultured in 24 -well plate at a concentration of $1 \mathrm{x} 106 \mathrm{cells} / \mathrm{ml}$ and incubated at $370 \mathrm{C}$ in a in a water saturated air atmosphere with 95 per cent humidity, 5 per cent $\mathrm{CO} 2$ (CO2 incubator). The supernatants after $48 \mathrm{~h}$ of infection were tested for the cytokines. (IFN $\gamma$ and IL4 level) by ELISA. The ELISA kits used were purchased from Quantikine R \& D (Minneopolis, USA) for quantitative estimation of cytokine levels of IL4 and IFN $\gamma$.

\section{Statistical analysis:-}

All experiments were carried out in triplicate with two sets of observations taken in each case. All data were analyzed for statistical significance using the Student's t-test.

\section{Results:-}

As illustrated in Table-5, A very high absolute CD3 cell count was observed in thyroid cases under both category segregated based on TSH value compared to HIV and VL patients or controls. No significant difference was observed for CD4 cell count in both categories of thyroid patients, however unlike to other reports, a reduction in CD4 count was very much apparent than healthy control but it was similar to identical reduced CD4 count observed in VL cases. The CD8+ T cell counts showed varied patterns in higher TSH category of thyroid patients and recorded a higher value whereas it was lower in the thyroid patients showing lower TSH value. Our results is in contrary to previous report where Both CD4+ and CD8+ T cells were reported to occur in thyroid lymphocytic infiltrate with a preponderance of CD4+ cells. As the present investigation was done on peripheral blood samples rather than lymphocyte infiltrate, it can be speculated that although CD4 $\mathrm{T}$ cells effectively operated in infiltrate, however a seizable proportion of CD8 $+\mathrm{T}$ cells migrate towards periphery to restrict protective factors influencing regulation of the infection and control of patients.

Subsequent results revealed that there was an increase in activated T-cell expressing markers like interferongamma. All hyper thyrodism patients had much higher interferon-gamma( IFN- $\gamma$ ) compared to Thyroid patients with low TSH range. Despite that, when compared from IFN value in VL and HIV cases, IFN- $\gamma$ produced in thyroid patient was much increased. VL patients and HIV in particular produced a low production of IFN- $\gamma$ and in this context, both were immuno suppressed. IFN- $\gamma$, usually produced by CD4 cells is key mediator given its ability to promote cellular immune activation in human VL patients. However apart from it, we observed a lower production of interleukin-4 in thyroid patients compared to VL cases and HIV but thyroid patients under hyper-thyroisism category were shown with the elevation in the levels of IL-4 which was about two-fold higher than in the thyroid cases with hypo-thyroidism state. Weetman et al(2000) have earlier demonstrated an increase in IL-4 cytokine in thyroid patients. However, since our study was done after segregating patients as under severe and moderate category, one can speculate that there occurs an increase in activated $\mathrm{T}$ cells particulary when a thyroid patient gets converted into advanced state. 
This study was conducted in peripheral blood samples to identify peripheral compartments of markers for this auto immune disease. These patients did not have any chronic disease and hence the abnormalities can be important to to correlate immunological abnormalities to the manifestation of thyroid disease

Alteration in Immune Response pattern at T-cell Level during hyper-thyroidism and hypothyroidism clinical condition of auto immune disorder in context to healthy control.

\begin{tabular}{|c|c|c|c|c|c|}
\hline \multirow{2}{*}{ Category } & \multicolumn{3}{|c|}{ (Absolute count $/ \mu \mathrm{I})$} & \multicolumn{2}{|c|}{ Cytokines (\%) } \\
\hline & CD-3 & CD-4 & CD-8 & IFN & IL-4 \\
\hline \multicolumn{6}{|l|}{ Auto immune disorder } \\
\hline \multicolumn{6}{|l|}{ 1. Thyroid Cases } \\
\hline Hyperthyroidism & $2848.3 \pm 20.72$ & $428.1 \pm 17.65$ & $421.1 \pm 22.1$ & $173.55 \pm 27.51$ & $66.95 \pm 8.51$ \\
\hline TSH Range & $(633-973)$ & $(283-603)$ & (263.4-604) & & \\
\hline \multicolumn{6}{|l|}{$(48.55+10.07$} \\
\hline Hypothyroidism & $3876.7 \pm 16.65$ & $489.3 \pm 34.2$ & $387.4 \pm 34.2$ & $138.37 \pm 27.31$ & $33.27 \pm 7.91$ \\
\hline (TSH Range- $(0.22-0.02)$ & 798.6-958 & $359-587$ & $(254-530)$ & & \\
\hline \multicolumn{6}{|l|}{$0.22+0.02$} \\
\hline \multicolumn{6}{|l|}{ OTHER DISEASE } \\
\hline $\mathrm{VL}(\mathrm{n}=10)$ & $2200 \pm 175.70$ & $453 \pm 265.80$ & $222 \pm 96.85$ & $135.80 \pm 36.92$ & $104.235 \pm 36.291$ \\
\hline HIV (n=10) & & $325.1 \pm 254.09$ & 1304.1683 .40 & $174.1 \pm 127.3$ & $174.00 \pm 69.27$ \\
\hline Control & $1385.00 \pm 253.80$ & $1099+429.06$ & $653 \pm 287.58$ & $105.22 \pm 4.65$ & $16.387 \pm 0.358$ \\
\hline
\end{tabular}

\section{Acknowledgement:-}

We are indebted to Director, RMRIMs for the permission

\section{References:-}

1. V. Kumar, "24: The endocrine system," in Robbins and Cotran Pathologic Mechanisms of Disease, p. 1113, Elsevier, Philidelphia, Pa, USA, 8th edition, 2010.

2. A. P. Weetman and A. M. McGregor, "Autoimmune thyroid disease: further developments in our understanding," Endocrine Reviews, vol. 15, no. 6, pp. 788-830, 1994.

3. K. Eguchi, N. Matsuoka, and S. Nagataki, "Cellular immunity in autoimmune thyroid disease," Bailliere's Clinical Endocrinology and Metabolism, vol. 9, no. 1, pp. 71-94, 1995.

4. A. Huber, F. Menconi, S. Corathers, E. M. Jacobson, and Y. Tomer, "Joint genetic susceptibility to type 1 diabetes and autoimmune thyroiditis: from epidemiology to mechanisms," Endocrine Reviews, vol. 29, no. 6, pp. 697-725, 2008.

5. M. A. Iddah, B. N. Macharia, A. G. Ng'wena, A. Keter, and A. V. O. Ofulla, "Thryroid hormones and hematological indices levels in thyroid disorders patients at Moi teaching and referral hospital, Western Kenya," ISRN Endocrinology, vol. 2013, Article ID 385940, 6 pages, 2013.

6. G. J. Canaris, N. R. Manowitz, G. Mayor, and E. C. Ridgway, "The Colorado thyroid disease prevalence study," Archives of Internal Medicine, vol. 160, no. 4, pp. 526-534, 2000.

7. S. M. McLachlan and B. Rapoport, "The molecular biology of thyroid peroxidase: cloning, expression and role as autoantigen in autoimmune thyroid disease," Endocrine Reviews, vol. 13, no. 2, pp. 192-206, 1992.

8. A. P. Weetman, "Endocrinology," in Handbook of HLA and Disease, R. I. Lechler and A. Warrens, Eds., Academic Press, London, UK, 2nd edition, 2000. 